\title{
Carbonyl Reductase 1 Plays a Significant Role in Converting Doxo- rubicin to Cardiotoxic Doxorubicinol in Mouse Liver, but the Majority of the Doxorubicinol-Forming Activity Remains Unidentified ${ }^{\mathbb{}}$
}

\author{
Daniel H. Breysse, Ryan M. Boone, ${ }^{1}$ Cameron M. Long, ${ }^{2}$ Miranda E. Merrill, ${ }^{3}$ \\ Christopher M. Schaupp, ${ }^{4}$ Collin C. White, Terrance J. Kavanagh, Edward E. Schmidt, \\ and Gary F. Merrill
}

Department of Biochemistry and Biophysics, Oregon State University, Corvallis, Oregon (D.H.B., R.M.B., C.M.L., M.E.M., G.F.M.); Department of Environmental and Occupational Health Sciences, University of Washington, Seattle, Washington (C.M.S., C.C.W., T.J.K.); and Department of Microbiology and Immunology, Montana State University, Bozeman, Montana (E.E.S.)

Received September 10, 2019; accepted December 23, 2019

\begin{abstract}
Doxorubicin is a widely used cancer therapeutic, but its effectiveness is limited by cardiotoxic side effects. Evidence suggests cardiotoxicity is due not to doxorubicin, but rather its metabolite, doxorubicinol. Identification of the enzymes responsible for doxorubicinol formation is important in developing strategies to prevent cardiotoxicity. In this study, the contributions of three murine candidate enzymes to doxorubicinol formation were evaluated: carbonyl reductase (Cbr) 1 , Cbr3, and thioredoxin reductase 1 (Tr1). Analyses with purified proteins revealed that all three enzymes catalyzed doxorubicindependent NADPH oxidation, but only Cbr1 and Cbr3 catalyzed doxorubicinol formation. Doxorubicin-dependent NADPH oxidation by Tr1 was likely due to redox cycling. Subcellular fractionation results showed that doxorubicin-dependent redox cycling activity was primarily microsomal, whereas doxorubicinol-forming activity was exclusively cytosolic, as were all three enzymes. An immunoclearing approach was used to assess the contributions of the three enzymes to doxorubicinol formation in the complex milieu of the cytosol. Immunoclearing Cbr1 eliminated $25 \%$ of the total doxorubicinol-forming activity in cytosol, but immunoclearing Cbr3 had no effect, even in Tr1 null livers that overexpressed Cbr3.
\end{abstract}

The immunoclearing results constituted strong evidence that Cbr1 contributed to doxorubicinol formation in mouse liver but that enzymes other than Cbr1 also played a role, a conclusion supported by ammonium sulfate fractionation results, which showed that doxorubicinol-forming activity was found in fractions that contained little Cbr1. In conclusion, the results show that Cbr1 accounts for $25 \%$ of the doxorubicinol-forming activity in mouse liver cytosol but that the majority of the doxorubicinolforming activity remains unidentified.

\section{SIGNIFICANCE STATEMENT}

Earlier studies suggested carbonyl reductase (Cbr) 1 plays a dominant role in converting chemotherapeutic doxorubicin to cardiotoxic doxorubicinol, but a new immunoclearing approach described herein shows that Cbr1 accounts for only $\mathbf{2 5 \%}$ of the doxorubicinol-forming activity in mouse liver cytosol, that two other candidate enzymes$\mathrm{Cbr} 3$ and thioredoxin reductase 1-play no role, and that the majority of the activity remains unidentified. Thus, targeting Cbr1 is necessary but not sufficient to eliminate doxorubicinol-associated cardiotoxicity; identification of the additional doxorubicinol-forming activity is an important next challenge.
G.F.M. was supported by an Oregon Health Sciences University Medical Research Foundation grant and an Oregon State University General Research Fund small grant. E.E.S. was supported by US National Institutes of Health National Cancer Institute [Grants CA152559-R21 and CA215784-RO1] and National Institute on Aging [Grants AG040020-RO1 and AG055022-R21], the Montana Agricultural Experiment Station [Grant MONB00443], and the Montana State University Department of Microbiology \& Immunology.

${ }^{1}$ Current affiliation: Oregon Health Sciences University, Portland, Oregon

${ }^{2}$ Current affiliation: Rochester, Minnesota.

${ }^{3}$ Current affiliation: Oregon Health Sciences University, Portland, Oregon

${ }^{4}$ Current affiliation: Neptune and Company, Lakewood, Colorado.

https://doi.org/10.1124/dmd.119.089326.

S This article has supplemental material available at dmd.aspetjournals.org.
Introduction

Doxorubicin (Dox), also known as Adriamycin, is used to treat a wide array of cancers, but its effectiveness is limited by dose-dependent cardiotoxicity, which manifests as congestive heart failure (Singal and Iliskovic, 1998; Swain et al., 2003; Kremer and Caron, 2004). The mechanism underlying cardiotoxicity is not fully understood, but doxorubicinol (Doxol), a metabolite generated by reduction of Dox at the C-13 carbonyl position (Fig. 1A) (Joerger et al., 2005) likely plays a significant role, as Doxol is far more potent than Dox in inhibiting several cardiac ion transporters (Boucek et al., 1987; Olson et al., 1988; Dodd et al., 1993; Hanna et al., 2014), and Doxol causes systolic and diastolic dysfunction in isolated rabbit heart muscle (Boucek et al., 1987; Olson et al., 1988).

ABBREVIATIONS: $11 \beta$-HSD1, $11 \beta$-hydroxysteroid dehydrogenase 1; Akr, aldo-keto reductase; Cbr, carbonyl reductase; Dox, doxorubicin; Doxol, doxorubicinol; Gclm, $\gamma$-glutamyl-cysteine ligase modulatory subunit; hydroxy-PP-Me, 3-(7-isopropyl-4-(methylamino)-7H-pyrrolo[2,3-d]pyrimidin5yl)phenol; LC-MS/MS, liquid chromatography-mass spectrometry/collision-induced dissociation mass spectrometry; NFDM, nonfat dried milk; Tr1, thioredoxin reductase 1; Trx1, thioredoxin1. 


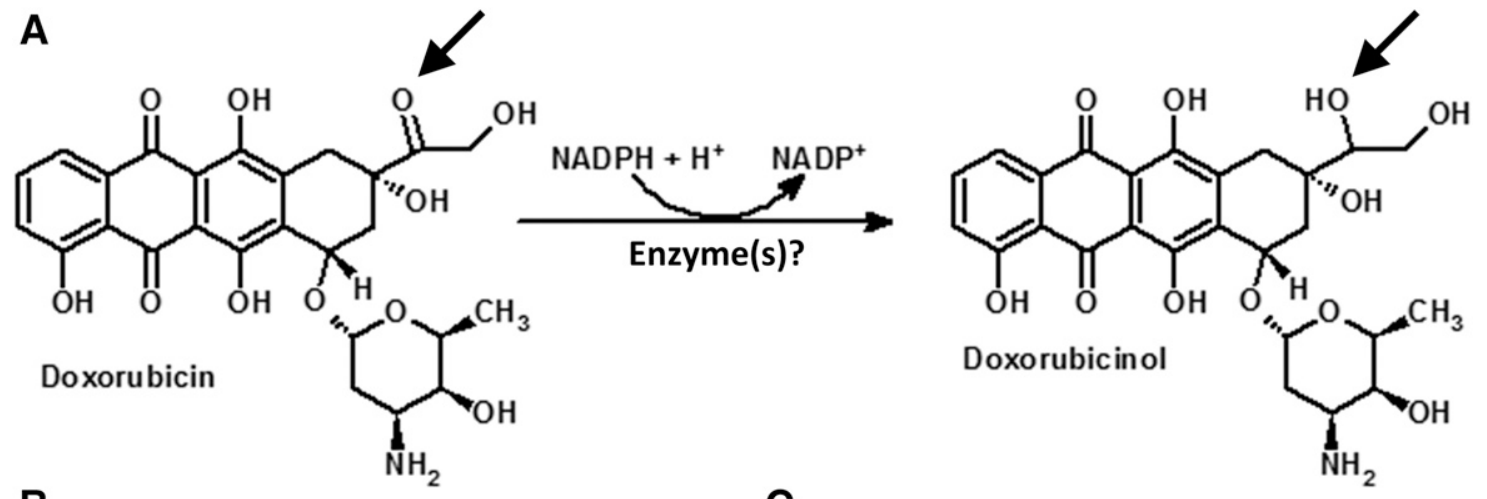

B

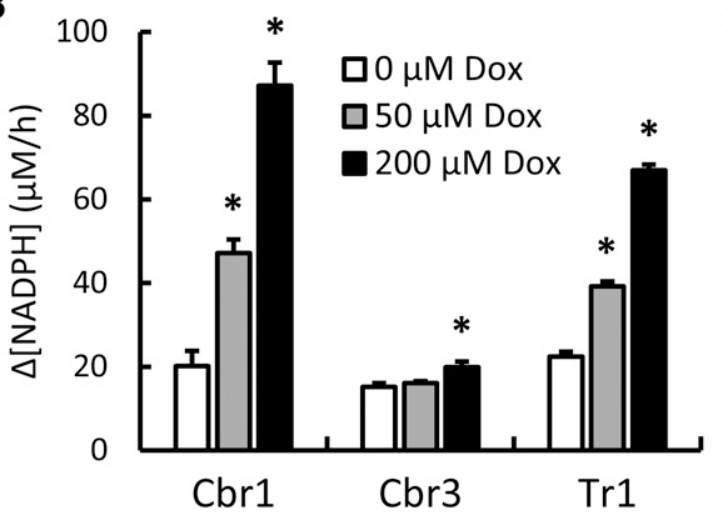

C

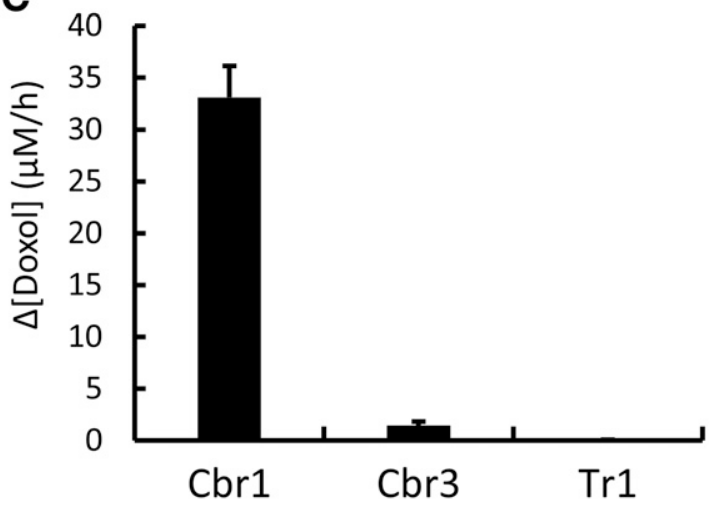

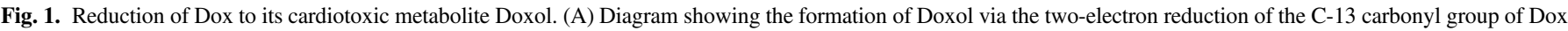

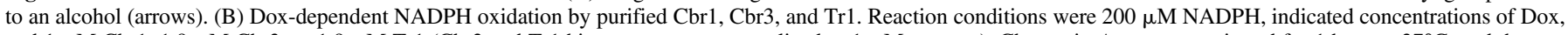

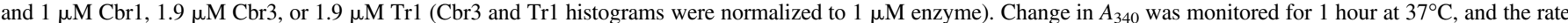

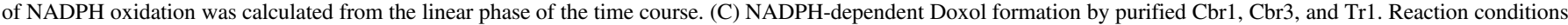

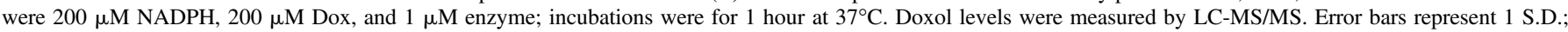
$n=3$ for all reactions; $* P<0.05$ by Student's $t$ test.

Compared with its parent compound, Doxol displays minimal antineoplastic activity (Olson et al., 1988; Chang et al., 1989; Bernardini et al., 1991; Heibein et al., 2012). This is critical, as it suggests that the antitumor properties of Dox are not inherently linked to its cardiotoxicity. Preventing the metabolism of Dox to Doxol could thus increase the safety of the drug while retaining its effectiveness. As such, the goal of this study was to directly assess the contributions of three cytosolic enzymes to Doxol formation in liver, the most dominant location of xenobiotic metabolism. The enzymes carbonyl reductase (Cbr) 1, Cbr3, and thioredoxin reductase 1 ( Tr1) were selected for the following reasons.

Cbr1 can catalyze the NADPH-dependent reduction of Dox to Doxol in vitro, and drugs that inhibit purified Cbr1 activity also inhibit Doxol formation by liver cytosol (Kassner et al., 2008; Bains et al., 2010), suggesting Cbr1 is the predominant Dox reductase in liver cytosol. Genetic evidence also suggests Cbr1 plays a major role in Doxol formation. Mice with a single null allele of Cbr1 show decreased sensitivity to Dox-induced cardiotoxicity, whereas mice that overexpress Cbr1 show increased sensitivity (Forrest et al., 2000; Olson et al., 2003). Additionally, cancer cell lines that overexpress carbonyl reductase are resistant to the antiproliferative effects of Dox (Bains et al., 2013). Pharmacological and genetic studies alone, however, do not confirm a major role for Cbr1 in Doxol formation, as drugs are often nonspecific and genetic interventions often have secondary effects on gene expression.

Cbr3 is a syntenic homolog of Cbr1. Although less well studied, it has also been shown to catalyze Doxol formation in vitro (Blanco et al., 2008; Bains et al., 2010). In vivo evidence for Cbr3 playing a role in Doxol formation derives from work done on knockout mice missing the $\gamma$-glutamyl-cysteine ligase modulatory subunit $(\mathrm{Gclm})$ gene encoding the glutamate-cysteine ligase modifier subunit. These mice overexpress Cbr3 mRNA, and to a lesser extent Cbr1 mRNA (Haque et al., 2010), and show elevated formation of Doxol by liver cytosol and isolated hepatocytes (Schaupp et al., 2015).

$\operatorname{Tr} 1$ is known to reduce many substrates besides thioredoxin, including the small molecules lipoate (Arnér et al., 1996), lipid hydroperoxides (Björnstedt et al., 1995), dehydroascorbate (May et al., 1997), and menadione (Luthman and Holmgren, 1982). Liver-specific deletion of the thioredoxin reductase 1 ( Txnrd1)gene encoding $\operatorname{Tr} 1$ also causes massive overexpression of Cbr3 (Bondareva et al., 2007; Suvorova et al., 2009), potentially as a compensatory response to the loss of $\operatorname{Tr} 1$. Because of its broad substrate specificity, as well as a potential substrate overlap with $\mathrm{Cbr} 3$, we evaluated $\operatorname{Tr} 1$ as another candidate for playing a role in Doxol formation.

The aldo-keto reductase (AKR) 1C3 can catalyze Doxol formation in vitro; however, we did not include it in our investigations because the inhibition profile of AKR1C3 does not match that of liver cytosol (Tanaka et al., 2005; Kassner et al., 2008).

Initially, purified recombinant enzymes were tested for their ability to catalyze Dox-dependent NADPH oxidation and NADPH-dependent Doxol formation in vitro. Although all three enzymes catalyzed Dox-dependent NADPH oxidation, only Cbr1 and Cbr3 catalyzed Doxol formation. Instead of Doxol formation, Tr1 catalyzed Dox-dependent generation of $\mathrm{H}_{2} \mathrm{O}_{2}$, probably via redox cycling. To directly test for a physiologic role of the three enzymes in Dox metabolism, antibodies to the three proteins were developed and used to immunoclear the respective proteins from liver cytosol. Immunoclearing Cbr3 or Tr1 had no effect on Doxol formation by liver cytosol, suggesting these enzymes 
played no physiologic role in Doxol formation. In contrast, immunoclearing Cbr1 eliminated $25 \%$ of the Doxol-forming activity, verifying that Cbr1 contributes to Doxol formation in vivo. Importantly, however, the results also showed that most of the Doxol-forming activity in liver cytosol was not Cbr1.

\section{Materials and Methods}

Mouse Liver Fractionation. Mice were handled using procedures approved by Oregon State University Institutional Animal Care and Use Committee. All mice were strain C57B16. A mixture of male and female mice between 3 and 9 months of age were used. No sex- or age-specific trends in the results obtained were observed. The $\Delta G c l m$ knockout line and liver-specific $\Delta T x n r d 1$ knockout line were described previously (Bondareva et al., 2007; Haque et al., 2010). Mice were fasted overnight to minimize variability caused by recent feeding activity and euthanized by cervical dislocation. Livers were perfused in situ with PBS $\left(150 \mathrm{mM} \mathrm{NaCl}, 50 \mathrm{mM} \mathrm{NaPO}_{4} \mathrm{pH}\right.$ 7.4) to reduce blood content, removed to a petri dish, minced, and homogenized (Elvehjem, five strokes) in 10 volumes cold MOPS isolation buffer $(200 \mathrm{mM}$ sucrose, $10 \mathrm{mM}$ Tris/4-morpholinepropanesulfonic acid pH 7.4, 1 mM EGTA) (Frezza et al., 2007). Homogenates were centrifuged for 10 minutes at $12,000 \mathrm{~g}$ to remove nuclei and undisrupted cells. Clarified lysates were centrifuged for 30 minutes at $45,000 \mathrm{~g}$ to remove mitochondria. The $45,000 \mathrm{~g}$ supernatants were centrifuged for 1 hour at $100,000 \mathrm{~g}$ to obtain microsomes $(100,000 \mathrm{~g}$ pellet) and cytosols $(100,000 \mathrm{~g}$ supernatant). Microsomes were resuspended in MOPS isolation buffer. Protein concentrations were determined by the Bradford method using bovine serum albumin as standard.

Ammonium sulfate fractionation was initiated by adding solid $\left(\mathrm{NH}_{4}\right)_{2} \mathrm{SO}_{4}$ to cytosol prepared from 13 pooled mouse livers to achieve a salt solution at $40 \%$ of saturation $(1.64 \mathrm{M})$. After stirring 30 minutes at $4{ }^{\circ} \mathrm{C}$, the mixture was centrifuged for 30 minutes at $12,000 \mathrm{~g}$ to obtain a $40 \%$ pellet and $40 \%$ supernatant. Solid $\left(\mathrm{NH}_{4}\right)_{2} \mathrm{SO}_{4}$ was added to the $40 \%$ supernatant, and the above steps were repeated to achieve first a $50 \%$ pellet and supernatant and subsequently a $60 \%$ pellet and supernatant. Precipitates were resuspended in $150 \mathrm{mM} \mathrm{KCl,} 50 \mathrm{mM} \mathrm{KPO}_{4} \mathrm{pH} 7.4$. Fractions were stored at $-20^{\circ} \mathrm{C}$ until assay.

Protein Expression and Purification. Using cDNA plasmids purchased from Open Biosystems (Huntsville, AL) as templates, polymerase chain reaction fragments containing the complete coding regions of mouse $\mathrm{Cbr} 1, \mathrm{Cbr} 3$, and $\operatorname{Tr} 1$ and a synthetic Nde1 site overlapping the start codon were blunt end-cloned into EcoR5-cleaved pBluescript KS and subcloned as Nde1/Hind3 fragments into the expression vector pET28a (Novagen, La Jolla, CA) such that expressed proteins had a histidine tag and thrombin cleavage site at the $\mathrm{N}$ terminus and the native amino acid at the $\mathrm{C}$ terminus. BL21 bacteria transformed with the expression plasmids were shaken at $37^{\circ} \mathrm{C}$ in LB medium containing $50 \mu \mathrm{g} / \mathrm{ml}$ kanamycin. When absorbance at $600 \mathrm{~nm}\left(A_{600}\right)$ reached $0.6,1 \mathrm{mM}$ isopropyl- $\beta$-D-thiogalactoside was added to induce protein expression, and incubation continued for 6 hours at $25^{\circ} \mathrm{C}$ or overnight at $18^{\circ} \mathrm{C}$. Cells were harvested by centrifugation at $3300 \mathrm{~g}$, resuspended in $20 \mathrm{ml}$ chilled extraction buffer $\left(150 \mathrm{mM} \mathrm{NaCl}, 50 \mathrm{mM} \mathrm{NaPO}_{4} \mathrm{pH}\right.$ 7.4), and lysed using an M-110P Microfluidizer (Microfluidics, Westwood, MA). Lysates were clarified by centrifugation at $15,000 \mathrm{rpm}$ for 30 minutes in a Sorvall SS-34 rotor. Clarified lysate was mixed with $1 \mathrm{ml}$ TALON metal affinity resin (Clontech, Mountain View, CA), and the mixture incubated for 1 hour with tumbling at $4{ }^{\circ} \mathrm{C}$. Resin containing bound protein was collected by centrifugation for 1 minute at $2000 \mathrm{~g}$, washed four times with $40 \mathrm{ml}$ chilled extraction buffer, transferred to a gravity column, and washed with $10 \mathrm{ml}$ extraction buffer and $5 \mathrm{ml}$ of extraction buffer with $5 \mathrm{mM}$ imidazole. Bound protein was eluted in $500-\mu 1$ increments using $5 \mathrm{ml}$ elution buffer (extraction buffer with $50 \mathrm{mM}$ imidazole). Protein concentration was determined by $A_{280}$ using extinction coefficients of $20,970 \mathrm{M}^{-1} \mathrm{~cm}^{-1}$ for $\mathrm{Cbr} 1$ and $\mathrm{Cbr} 3$ and $58,330 \mathrm{M}^{-1} \mathrm{~cm}^{-1}$ for Tr1. Protein purity, assessed by SDS-PAGE and Coomassie staining, was greater than 95\%; thus no further purification was required. Gels representative of the purity achieved are shown in Supplemental Material (Supplemental Fig. 1). Proteins were stored at $4^{\circ} \mathrm{C}$ in elution buffer containing $0.1 \mathrm{mM}$ dithiolthreitol. Freeze/thawing or storage at concentrations above $25 \mu \mathrm{M}$ led to protein precipitation and was avoided. When precipitation occurred, fresh preparations of enzyme were made.

Coupling of Cbr1 and Cbr3 to Affi-gel 15 and Tr1 to Affi-gel 10 was done using the aqueous protocol described by the vendor (Bio-Rad, Hercules, CA).
Prior to coupling, imidazole and dithiolthreitol were removed by gel filtration using $150 \mathrm{mM} \mathrm{KCl}, 50 \mathrm{mM} \mathrm{KPO}_{4} \mathrm{pH} 8.0$ as exchange buffer.

Immunologic Procedures. Antisera were generated in New Zealand White rabbits, using full-length mouse $\mathrm{Cbr} 1, \mathrm{Cbr} 3$, and $\mathrm{Tr} 1$ protein with N-terminal histidine tags as antigen, expressed and purified as described above. Cbr3 and Tr1 immunizations and bleeds were done at the Oregon State University Laboratory Animal Resources Center. Cbr1 immunizations and bleeds were done by Pacific Immunology (Ramona, CA). Two rabbits were used for each protein. Antisera identification codes were assigned based on the source rabbit (Cbr1, 12081 and 12082; Cbr3, OB2 and OB4; Tr1, OB1 and OB3). Terminal bleeds (100-120 ml) were done by heart puncture. Raw antisera were prepared by allowing blood to coagulate at $4^{\circ} \mathrm{C}$ overnight, centrifuging the mixture at $5000 \mathrm{~g}$ for 15 minutes, and storing $10-\mathrm{ml}$ aliquots of supernatant at $-70^{\circ} \mathrm{C}$. Woring aliquots were stored at $4^{\circ} \mathrm{C}$, with $0.1 \% \mathrm{NaN}_{3}$ added as a preservative.

Raw Cbr3 antiserum cross-reacted with Cbr1 and raw Cbr1 antiserum crossreacted with $\mathrm{Cbr} 3$ when used to probe immunoblots. To increase specificity, raw Cbr1 antiserum (12081) was cross-adsorbed to Cbr3-conjugated Affi-gel 15 (Bio-Rad) to obtain Cbr1-specific antiserum, and raw Cbr3 antiserum (OB4) was cross-adsorbed to Cbr1-conjugated Affi-gel to obtain Cbr3-specific antiserum. Comparison of immunoblots probed with raw $\mathrm{Cbr} 3$ antiserum, Cbr3-specific antiserum, and Cbr1-specific antiserum showed that Cbr1 and Cbr3 migrated as a triplet during SDS-PAGE analysis of mouse liver cytosol, with the slower two bands representing Cbrl and the faster band representing Cbr3.

To remove bulk immunoglobulins that would compete for Protein A binding during immunoclearing, Cbr1- and Cbr3-specific antisera were affinity-purified. Briefly, $10 \mathrm{ml}$ of specific antisera were incubated with $5 \mathrm{ml}$ of Cbr1-conjugated or Cbr3-conjugated Affi-gel 15 beads overnight at $4^{\circ} \mathrm{C}$, the beads were rinsed several times with PBS, and bound antibodies were eluted with $150 \mathrm{mM} \mathrm{NaCl}, 20 \mathrm{mM}$ $\mathrm{HCl}$ into tubes containing $100 \mu \mathrm{l}$ of neutralizing $1 \mathrm{M} \mathrm{KPO}_{4} \mathrm{pH} 7.5$ per $1.5 \mathrm{ml}$ of eluate.

Immunoclearing of $\mathrm{Cbr} 1$ or $\mathrm{Cbr} 3$ was done using a noncovalent bridge of affinity-purified Cbr1-specific or Cbr3-specific antibody bound to Protein A magnetic beads (Bio-Rad). Beads were preincubated overnight with antibodies and washed several times with PBS immediately before use. Beads preincubated only with PBS were used for mock-cleared controls. About $50 \mu 1$ of washed beads was used to clear Cbr1 or Cbr3 from $120 \mu \mathrm{g}$ of cytosolic liver protein.

Immunoclearing of $\operatorname{Tr} 1$ was done using affinity-purified anti-Tr1 antibody that was covalently coupled to Affi-gel 10. Covalent coupling was necessary because the molecular weights of $\operatorname{Tr} 1$ and IgG heavy chain are similar $(50 \mathrm{kDa})$ and unavoidable leaching of some $\mathrm{IgG}$ using the noncovalent Protein A bridge method would complicate interpretation of immunoblots designed to measure the efficiency of Tr1 removal. Ethanolamine-coupled Affi-gel was used for mockcleared controls. About $50 \mu \mathrm{l}$ of anti-Tr1-coupled Affi-gel was used to clear $\operatorname{Tr} 1$ from $300 \mu \mathrm{g}$ of cytosolic liver protein.

After the efficiency of immunoclearing was determined by immunoblotting, $12-\mu \mathrm{g}$ equivalents of immunocleared or mock-cleared cytosolic protein were assayed for Dox-dependent NADPH oxidation activity and NADPH-dependent Doxol forming activity as described below.

For immunoblot analyses, proteins were separated by SDS-PAGE and electroblotted to nitrocellulose membrane (Bio-Rad). Membranes were blocked overnight at $4^{\circ} \mathrm{C}$ in TBST (20 mM Tris pH 7.4, $150 \mathrm{mM} \mathrm{NaCl}, 0.1 \%$ Tween) containing $5 \%$ nonfat dried milk (NFDM). Primary antibody incubations were for at least 2 hours at $25^{\circ} \mathrm{C}$, using a 1:1000 dilution of raw antiserum or 1:300 dilution of affinity-purified antibody in TBST 5\% NFDM. After four 5-minute washes with TBST 5\% NFDM, blots were incubated with secondary antibody for at least 1 hour at $25^{\circ} \mathrm{C}$, using a 1:5000 dilution of goat anti-rabbit horseradish peroxidase-conjugated IgG (Bio-Rad). Following four TBST washes, blots were developed with Western Lightning enhanced chemiluminescent substrate (Santa Cruz Biotechnology, Dallas, TX) and imaged using a ChemiGenius Bio-imaging system (Syngene, Frederick, MD). Protein band intensities were determined using Syngene Genetools software.

Enzyme Assays. Dox-dependent NADPH oxidation was measured in UV-transparent 96 -well plates by tracking $A_{340}$ using a Biotek Synergy 2 Multi-Mode Microplate Reader (Winooski, VT). Reaction cocktails contained $50 \mathrm{mM} \mathrm{KPO}_{4} \mathrm{pH} 7,0-200 \mu \mathrm{M}$ Dox, 1-2 $\mu \mathrm{M}$ recombinant enzyme or 50-100 $\mu \mathrm{g}$ of liver protein, and $200 \mu \mathrm{M}$ NADPH (added last) in a total volume of $150 \mu \mathrm{l}$. Absorbance was monitored for 1 hour at $37^{\circ} \mathrm{C}$, and the molar amount of NADPH 
TABLE 1

Kinetic parameters $\mathrm{Km}, \mathrm{k}_{\text {cat }}$, and $\mathrm{k}_{\mathrm{cat}} / \mathrm{Km}$ for Dox-dependent NADPH oxidation by purified $\mathrm{Cbr} 1, \mathrm{Cbr}$, and $\operatorname{Tr} 1$

\begin{tabular}{lcccc}
\hline \multirow{2}{*}{ Enzyme } & $R^{2}$ & \multicolumn{2}{c}{ Parameter } \\
\cline { 3 - 5 } & & $\mathrm{Km}$ & $\mathrm{k}_{\text {cat }}$ & $\mathrm{k}_{\text {cat }} / \mathrm{Km}$ \\
\hline & & $\mu \mathrm{M}$ & $\mathrm{s}^{-1} \times 10^{-3}$ & $\mu \mathrm{M}^{-1} \mathrm{~s}^{-1} \times 10^{-6}$ \\
Cbr1 & 0.989 & 130 & 15 & 120 \\
Cbr3 & 0.920 & 41 & 10 & 35 \\
Tr1 & 0.969 & 36 & 10 & 290 \\
\hline
\end{tabular}

oxidized was calculated using an extinction coefficient of $6220 \mathrm{M}^{-1} \mathrm{~cm}^{-1}$ and estimated pathlength of $3 \mathrm{~mm}$. The rate of NADPH oxidation in the absence of Dox was subtracted from rates measured in the presence of Dox.

NADPH-dependent Doxol formation was measured by liquid chromatography-mass spectrometry/collision-induced dissociation mass spectrometry (LC-MS/MS). Reaction cocktails were prepared as described above. After a 1-hour incubation at $37^{\circ} \mathrm{C}$, reactions were stopped and proteins precipitated by adding chilled sulfosalicylic acid to $0.5 \%$. Precipitated protein was removed by centrifugation for 10 minutes at $12,000 \mathrm{~g}$. Supernatants were analyzed using a SCIEX 3200 Q TRAP LC-MS/MS system equipped with Shimadzu LC-20AD pumps, SIL-20A model autosampler, CTO-10ASvp model column oven, and CBM-20A model system controller. Dox was separated from Doxol using an Agilent Poroshell 120 PFP reversed-phase column (Santa Clara, CA), $10 \mu$ l sample injection volume, $0.3 \mathrm{ml} / \mathrm{min}$ flow rate, and $10 \%-90 \%$ acetonitrile gradient applied over 10 minutes. For Dox, the Q1 parent peak was 544.4 $\mathrm{Da}$, and the Q3 peaks were 397.3, 379.3, and 130.1 Da. For Doxol, the Q1 parent peak was 546.4 Da, and Q3 peaks were 399.3, 381.3, and 130.1 Da. The 399.3 peak was used for quantitation of Doxol, and the other peaks were used as quality controls. Representative mass spectra are shown in Supplemental Material (Supplemental Fig. 2).

Kinetic parameters were calculated using nonlinear least squares regression. For mouse liver cytosol, $\mathrm{V}_{\max }$ was calculated instead of the catalytic constant $\left(\mathrm{k}_{\mathrm{cat}}\right.$ ). Raw data are provided in Supplemental Material (Supplemental Table 1).

$\mathrm{H}_{2} \mathrm{O}_{2}$ formation was measured using an Amplex red-based assay kit (Invitrogen, Carlsbad, CA). Purified Cbr1 and $\operatorname{Tr} 1(1 \mu \mathrm{M})$ were mixed with $50 \mu \mathrm{M}$ Dox in $50 \mathrm{mM} \mathrm{KPO}_{4} \mathrm{pH}$ 7.4. NADPH $(200 \mu \mathrm{M})$ was added to initiate the reaction and bring the final volume to $100 \mu$ l. Samples were combined with $100 \mu \mathrm{l}$ of Amplex red working solution $(100 \mu \mathrm{M}$ Amplex red, $200 \mathrm{U} / \mathrm{ml}$ horseradish peroxidase, $50 \mathrm{mM} \mathrm{KPO}_{4} \mathrm{pH}$ 7.4). Using a Qubit 3.0 fluorometer (Thermo Fisher Scientific, Waltham, MA), samples were excited at $470 \mathrm{~nm}$, and emission in the far-red range (665-720 nm) was tracked. A standard curve of 0 to $1 \mathrm{mM} \mathrm{H}_{2} \mathrm{O}_{2}$ was used to quantify $\mathrm{H}_{2} \mathrm{O}_{2}$ production by samples. The same procedure was followed to measure $\mathrm{H}_{2} \mathrm{O}_{2}$ production by $30 \mu \mathrm{g}$ of liver cytosolic or microsomal protein.

\section{Results}

Dox-Dependent NADPH Oxidation and NADPH-Dependent Doxol Formation by Purified Cbr1, Cbr3, and Tr1. As described above, the enzymes Cbr1, Cbr3, and Tr1 were potential candidates for catalyzing reduction of Dox to Doxol (Fig. 1A). As an initial assessment of these candidate enzymes, recombinant mouse Cbr1, Cbr3, and $\operatorname{Tr} 1$ were purified and tested for their ability to catalyze Dox-dependent NADPH oxidation. All three enzymes were active (Fig. 1B). Kinetic parameters for Dox-dependent NADPH oxidation by these enzymes were determined and are summarized in Table 1 . Cbr1 had a $\mathrm{k}_{\mathrm{cat}} 10$-fold greater than $\mathrm{Cbr} 3$ and 1.5-fold greater than $\operatorname{Tr} 1$. Tr1 had the highest catalytic efficiency $\left(\mathrm{k}_{\mathrm{cat}} / \mathrm{Km}\right)$.

Although spectrophotometric analysis of NADPH oxidation was an expedient way of assessing whether an oxidoreductase used Dox as a substrate, it did not establish that the product of the reaction was Doxol. For example, ring keto groups on Dox could alternatively serve as electron acceptors. We thus used LC-MS/MS to directly measure the production of Doxol by Cbr1, Cbr3, and Tr1. Pilot analyses with standards established that Doxol could be distinguished from its precursor and that the Doxol mass/charge $(\mathrm{M} / \mathrm{Z})$ peaks were proportional to the amount of Doxol added to the mixture (Supplemental Fig. 2). Using the LC-MS/MS assay, the amount of Doxol formed by each enzyme during a 1-hour incubation with $200 \mu \mathrm{M}$ NADPH and Dox was determined (Fig. 1C). Both Cbr1 and Cbr3 produced Doxol. In contrast, despite showing a high rate of Dox-dependent NADPH oxidation, $\operatorname{Tr} 1$ produced no Doxol during the reaction. Thus, tracking Dox-dependent NADPH oxidation alone was not sufficient to draw conclusions regarding the formation of Doxol. Kinetic parameters for Doxol formation by Cbr1 and Cbr3 were determined and compared with kinetic parameters for Doxol formation by mouse liver cytosol (Table 2). Cbr1 and Cbr3 had similar Km values (99 and $74 \mu \mathrm{M}$, respectively), but the catalytic efficiency $\left(\mathrm{k}_{\mathrm{cat}} / \mathrm{Km}\right)$ for Doxol formation by Cbr1 was 280 -fold higher than by Cbr3. Importantly, the $\mathrm{Km}$ values for Doxol formation exhibited by purified Cbr1 and Cbr3 were similar to those exhibited by liver cytosol $(75 \mu \mathrm{M})$, consistent with these enzymes playing a role in vivo.

Doxorubicin-Dependent Redox Cycling Catalyzed by Tr1. Because Dox-dependent NADPH oxidation by $\operatorname{Tr} 1$ was not associated with Doxol formation, we investigated whether $\operatorname{Tr} 1$ was catalyzing NADPH oxidation by a redox cycling mechanism (Fig. 2A). In this model, an electron equivalent from NADPH is transferred to Dox to form a semiquinone but is subsequently transferred to another entity, presumably solvent oxygen, which results in regeneration of Dox and the sequential formation of superoxide radical and $\mathrm{H}_{2} \mathrm{O}_{2}$. To test this hypothesis, Tr1 and Cbr1 were incubated with Dox and NADPH, and the amount of $\mathrm{H}_{2} \mathrm{O}_{2}$ produced over the course of the reaction was measured using Amplex Red reactivity as an indicator. The results (Fig. 2B) showed that in the presence of Dox and NADPH, Tr1

TABLE 2

Kinetic parameters $\mathrm{Km}, \mathrm{k}_{\text {cat }}$, and $\mathrm{k}_{\text {cat }} / \mathrm{Km}$ for NADPH-dependent Doxol formation by purified Cbr1 and Cbr3, as well as $\mathrm{Km}$ and $\mathrm{V}_{\text {max }}$ for NADPH-dependent Doxol formation by mouse liver cytosol

\begin{tabular}{|c|c|c|c|c|c|}
\hline \multirow{2}{*}{ Enzyme } & \multirow{2}{*}{$R^{2}$} & \multicolumn{4}{|c|}{ Parameter } \\
\hline & & $\mathrm{Km}$ & $\mathrm{k}_{\text {cat }}$ & $\mathrm{k}_{\mathrm{cat}} / \mathrm{Km}$ & $\mathrm{V}_{\max }$ \\
\hline & & $\mu \mathrm{M}$ & $s^{-1} \times 10^{-3}$ & $\mu \mathrm{M}^{-1} \mathrm{~s}^{-1} \times 10^{-6}$ & $\mu \mathrm{M} / \mathrm{h}$ \\
\hline Cbr1 & 0.997 & 99 & 36 & 360 & - \\
\hline Cbr3 & 0.855 & 74 & 0.1 & 1.3 & - \\
\hline Cytosol $(0.55 \mu \mathrm{g} / \mu \mathrm{l})$ & 0.976 & 75 & - & - & 8.7 \\
\hline
\end{tabular}




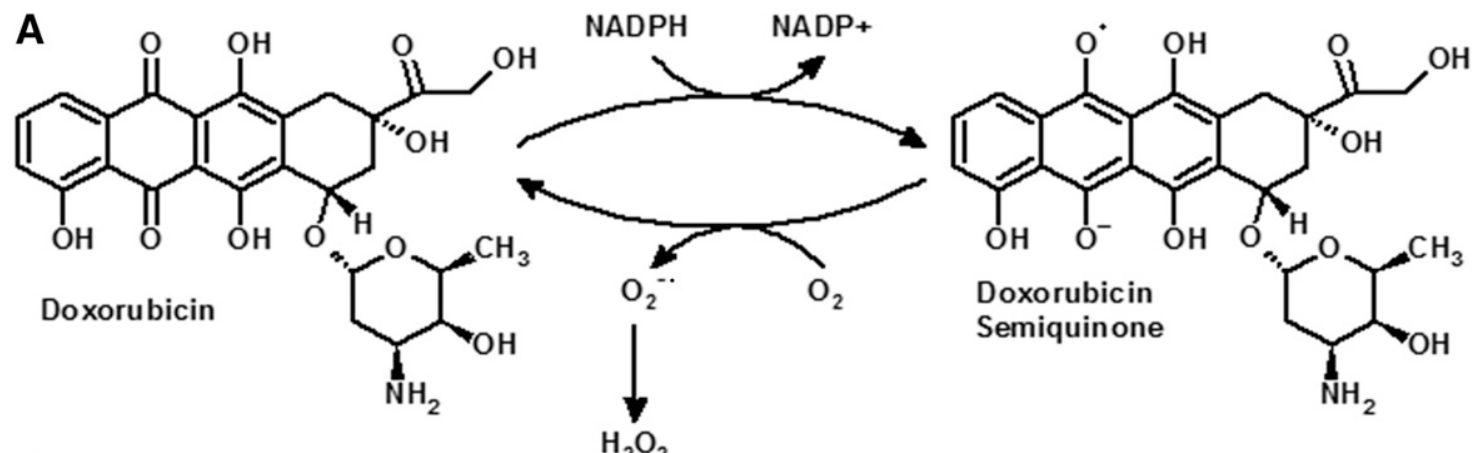

B

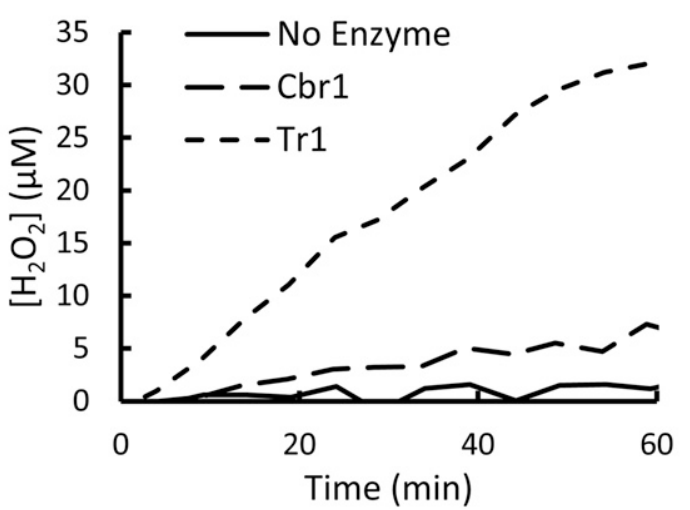

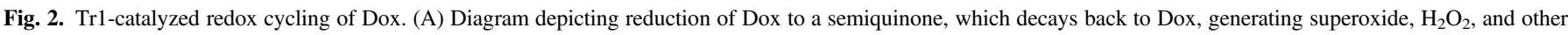

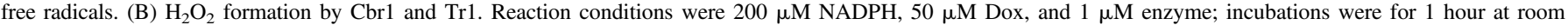
temperature.

generated significant amounts of $\mathrm{H}_{2} \mathrm{O}_{2}$. Cbrl also produced $\mathrm{H}_{2} \mathrm{O}_{2}$, but at a much slower rate. The efficient formation of $\mathrm{H}_{2} \mathrm{O}_{2}$, along with the lack of Doxol formation, suggested $\operatorname{Tr} 1$ was indeed catalyzing Dox-dependent redox cycling.

Subcellular Localization of Dox-Metabolizing Activities in Liver. To confirm the cytosolic localization of Cbr1, Cbr3, and $\operatorname{Tr} 1$ and to determine whether the enzymes catalyzing Dox-dependent NADPH oxidation and Doxol formation were similarly cytosolic, 45,000 $\mathrm{g}$ liver supernatants were fractionated into their microsomal and cytosolic components by centrifugation at $100,000 \mathrm{~g}$. Equal amounts of protein from each component were analyzed for $\mathrm{Cbr} 1, \mathrm{Cbr} 3$, and $\mathrm{Tr} 1$ protein by immunoblotting, Dox-dependent NADPH oxidation by spectrophotometry, and Doxol-forming activity by LC-MS/MS. Immunoblot analyses (Fig. 3A) confirmed that all three proteins were primarily cytosolic. However, when Dox-dependent NADPH oxidation activity was assayed, most of the activity present in the original $45,000 \mathrm{~g}$ supernatant was recovered in the microsomal fraction, and only $30 \%$ of the activity was recovered in the cytosolic fraction (Fig. 3B). In contrast, when Doxol formation was assayed, almost all of the activity present in the $45,000 \mathrm{~g}$ supernatant was recovered in the cytosolic fraction (Fig. 3C). To test whether the high levels of Dox-dependent NADPH oxidation activity observed in microsomal fractions was due to redox cycling, we measured the production of $\mathrm{H}_{2} \mathrm{O}_{2}$ by cytosolic and microsomal fractions. As shown in Fig. 3D, microsomal fractions produced significant amounts of $\mathrm{H}_{2} \mathrm{O}_{2}$, whereas cytosolic fractions did not, suggesting that Dox-dependent redox cycling was occurring in the microsomes.

Effect of Immunoclearing Cbr1 on Doxol-Forming Activity in Mouse Liver Cytosol. As demonstrated above, purified Cbr1 and Cbr3 were able to catalyze NADPH-dependent Doxol formation, with Cbr1 catalyzing the reaction much more efficiently than Cbr3. However, these in vitro analyses did not establish the extent to which these enzymes contributed to Doxol formation in the complicated milieu of the cytosol. Posttranslational modifications, accessory proteins, and small molecule effectors could impact the formation of Doxol by these enzymes in vivo. Additionally, other Doxol-forming enzymes might exist in the cytosol, diluting the contributions of Cbr1 and Cbr3 to overall activity. Genetic or drug interventions are often used to study the role of specific proteins within an organism or cell; however, genetic interventions often have unpredictable effects on the transcriptome, and "specific" drugs often target many enzymes in addition to the one being studied. We thus developed an immunoclearing approach to analyze the roles of Cbr1, Cbr3, and Tr1 in Doxol formation. This approach avoids complications that could arise from genetic or drug interventions while still requiring the enzymes to function within the context of the cytosol.

Protein A magnetic beads preincubated with Cbr1-specific antibody were used to immunoclear Cbr1 from six mouse liver cytosols. Beads preincubated only with buffer served as mock-cleared controls. The amount of Cbrl present in Cbr1-cleared and mock-cleared cytosols was determined by immunoblot analysis (Fig. 4A). Densitometry showed that on average $67 \%$ of Cbr1 was cleared. To confirm the specificity of the immunoclearing reaction, immunoblots were reprobed with antibody against the small redox protein thioredoxin (Trx1). As expected, there was no significant difference in the amount of Trx 1 present in Cbr1-cleared and mock-cleared cytosols (Fig. 4A).

Equivalent amounts of Cbr1-cleared and mock-cleared cytosolic protein were incubated with NADPH and Dox, and the amount of Doxol produced during a 1-hour reaction was measured by LC-MS/MS (Fig. 4B). Five of six cytosols showed a statistically significant reduction in the amount of Doxol produced by Cbr1-cleared cytosol, compared with mock-cleared cytosol. When averaged, Doxol formation by the Cbr1-cleared cytosols was $21 \%$ lower than by the mock-cleared 

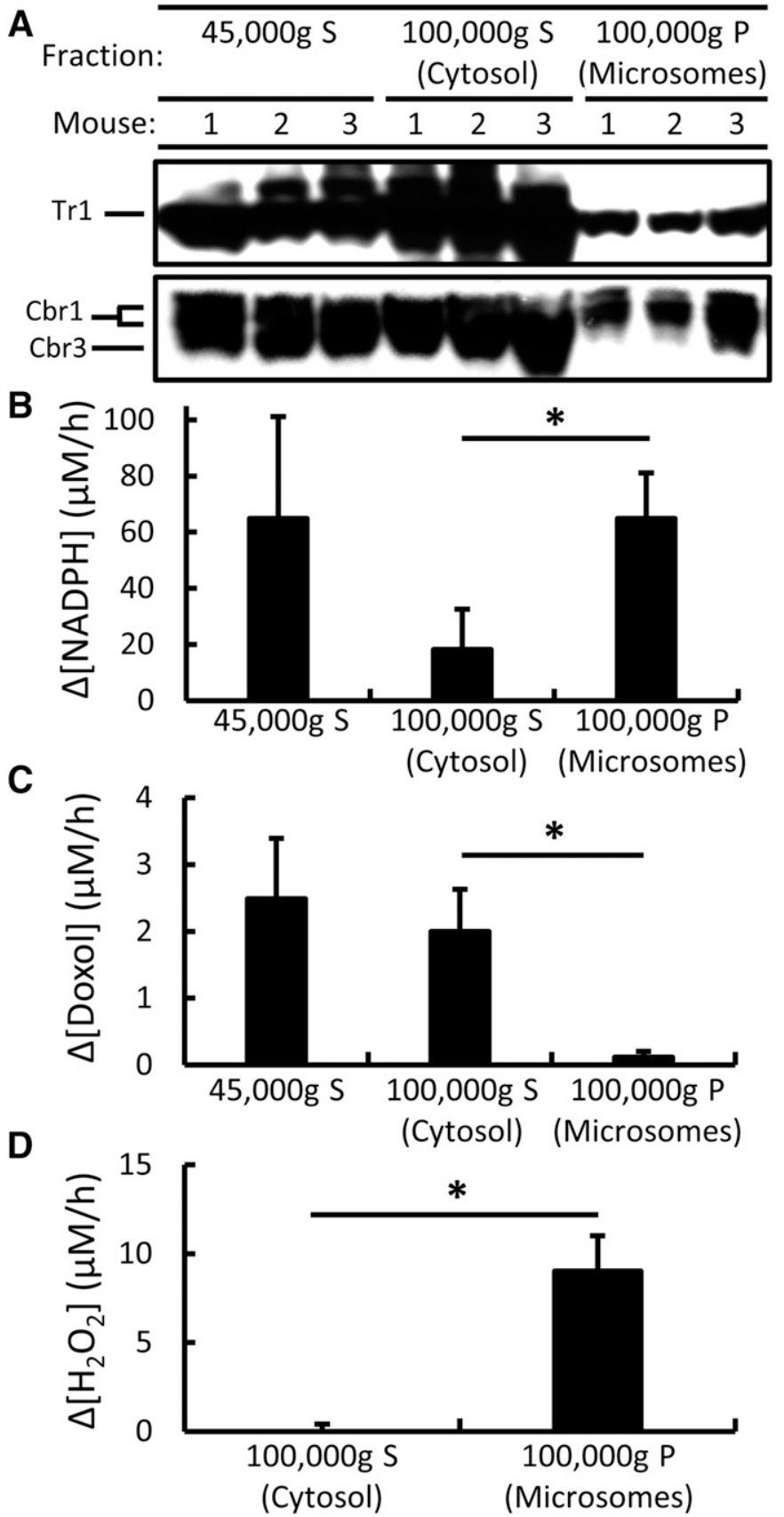

Fig. 3. Subcellular localization of Cbr1, Cbr3, and Tr1 protein; Dox-dependent NADPH oxidation activity; NADPH-dependent Doxol-forming activity; and Dox-dependent $\mathrm{H}_{2} \mathrm{O}_{2}$-forming activity in mouse liver. (A) Immunoblot analysis of $\mathrm{Cbr1}$, $\mathrm{Cbr} 3$, and $\operatorname{Tr} 1$ protein in indicated supernatants $(\mathrm{S})$ and pellets $(\mathrm{P}): 45,000 \mathrm{~g} \mathrm{~S}$ (a mixture of cytosol and microsomes), 100,000 $\mathrm{g}$ (cytosol), and 100,000 $\mathrm{g}$ (microsomes). $\Delta \mathrm{Gclm}$ lysates were used to facilitate Cbr3 localization. (B) Dox-dependent NADPH oxidation by indicated fractions of wild-type liver lysates. Reaction conditions were $200 \mu \mathrm{M}$ $\mathrm{NADPH}, 200 \mu \mathrm{M}$ Dox, and $50 \mu \mathrm{g}$ fraction protein; incubations were for 1 hour at $37^{\circ} \mathrm{C}$. (C) NADPH-dependent Doxol formation by indicated fractions of wild-type liver lysates. Reaction conditions and incubations were as described in (B). Doxol levels were measured by LC-MS/MS. (D) $\mathrm{H}_{2} \mathrm{O}_{2}$ formation by indicated fractions of wild-type liver lysates. Reaction conditions were $200 \mu \mathrm{M}$ NADPH, $50 \mu \mathrm{M}$ Dox, and $30 \mu \mathrm{g}$ fraction protein; incubations were for 1 hour at $25^{\circ} \mathrm{C}$. Error bars represent 1 S.D.; $n=3$ for all reactions; $* P<0.05$ by Student's $t$ test.

group (Fig. 4C). Because the immunoblotting analyses showed that the immunoclearing procedure removed only $67 \%$ of the Cbrl in the cytosol, we calculated that $31 \%$ of the Doxol-forming activity in mouse liver cytosol was attributable to Cbr1. When the immunoclearing analyses were repeated using the same cytosols and independently prepared new cytosols $(n=12)$, slightly different estimates for the amount of activity attributable to Cbr1 were obtained, ranging from an average of $20 \%$ to $31 \%$. From all experiments, we calculated that on average $23.4 \% \pm 13.7 \%$ of Doxol-forming activity (roughly $25 \%$ ) was attributable to Cbr1. The immunoclearing results thus provided unequivocal evidence that Cbr1 contributed significantly to Doxol formation in liver. Importantly, however, even with the majority of Cbr1 cleared from the cytosol, most of the Doxol-forming activity remained.

Effect of Immunoclearing Cbr3 and Tr1 on Dox-Dependent NADPH Oxidation and Doxol-Forming Activity in Mouse Liver Cytosol. The above results showed that Cbr1 accounted for about $25 \%$ of the total Doxol-forming activity in liver cytosol. To investigate whether Cbr3 and Tr1 contributed to Dox metabolism, these proteins were also selectively immunocleared from mouse liver cytosol. Immunoblot analyses showed that $\mathrm{Cbr} 3$ and $\operatorname{Tr} 1$ were efficiently removed by the immunoclearing procedure (Fig. 5A). Despite near total clearing of Cbr3, the levels of Doxol-forming activity were unaffected (Fig. 5, B and C). Thus, Cbr3 did not contribute to the Doxol-forming activity detected in liver cytosol. Dox-dependent NADPH oxidation activity was also measured in Tr1-cleared and mock-cleared samples, and no significant difference was observed (Fig. 5, D and E). Thus, although purified $\operatorname{Tr} 1$ efficiently catalyzed Dox-dependent NADPH oxidation in vitro, $\operatorname{Tr} 1$ did not contribute to Dox-dependent NADPH oxidation in the context of the cytosol.

Doxol Formation in $\boldsymbol{\Delta}$ Txnrd1 Mouse Liver Cytosol. Cbr3 was implicated as a potential Dox reductase when it was observed that $\Delta G c l m$ null mice, which have elevated levels of Cbr3 mRNA and protein, have higher rates of Doxol formation (Schaupp et al., 2015). Interestingly, liver-specific deletion of the Txnrdl gene also resulted in greatly elevated levels of $\mathrm{Cbr} 3$ and mildly elevated levels of Cbr1 (Fig. 6A). We investigated whether Cbr3 contributed significantly to Doxol formation in tissues that overexpressed Cbr3. We found that $\Delta$ Txnrd1 cytosols produced significantly more Doxol, but the effect was small (35\% increase), relative to the large increase in $\mathrm{Cbr} 3$ protein (Fig. 6A).

To ascertain whether the increased Doxol-forming activity observed in $\Delta$ Txnrd1 null cytosols was attributable to Cbr3, Cbr1, or both enzymes, we immunocleared $\mathrm{Cbr} 3$ and Cbr1 individually and simultaneously from four $\Delta T x n r d 1$ cytosols (Fig. 6C). Total clearing of Cbr3 was achieved in all cytosols, whereas clearing of Cbr1 was less thorough (on average, about 55\%). Clearing Cbr3 had no effect on Doxol formation, whereas clearing Cbr1 again led to a reduction in Doxol formation. Clearing Cbr1 and Cbr3 simultaneously did not lead to any difference in Doxol formation when compared with clearing Cbr1 alone (Fig. 6D). This suggests that even when Cbr3 was highly overexpressed, it was not responsible for any of the observed Doxol-forming activity in liver cytosol. The increased Doxol formation observed in $\Delta T x n r d 1$ livers was thus likely due to the small increase in Cbrl or some as yet unidentified enzyme.

Ammonium Sulfate Fractionation of Doxol-Forming Activity in Liver Cytosol. Having shown that only $25 \%$ of the Doxol-forming activity in liver cytosol was attributable to Cbr1, most of the total Doxol-forming activity remained unidentified. As an initial means of characterizing this remaining activity, we fractionated liver cytosol by ammonium sulfate precipitation. SDS-PAGE analysis revealed that each fraction had a distinct protein profile (Fig. 7A). Immunoblot analysis revealed that Cbr1 was found primarily in the $60 \%$ supernatant (Fig. 7B), whereas Doxol-forming activity was found primarily in the $60 \%$ pellet and $50 \%$ pellet (Fig. 7C). The finding that peak Doxol-forming activity was found in an ammonium sulfate fraction containing relatively little Cbrl independently corroborated the conclusion of the immunoclearing 

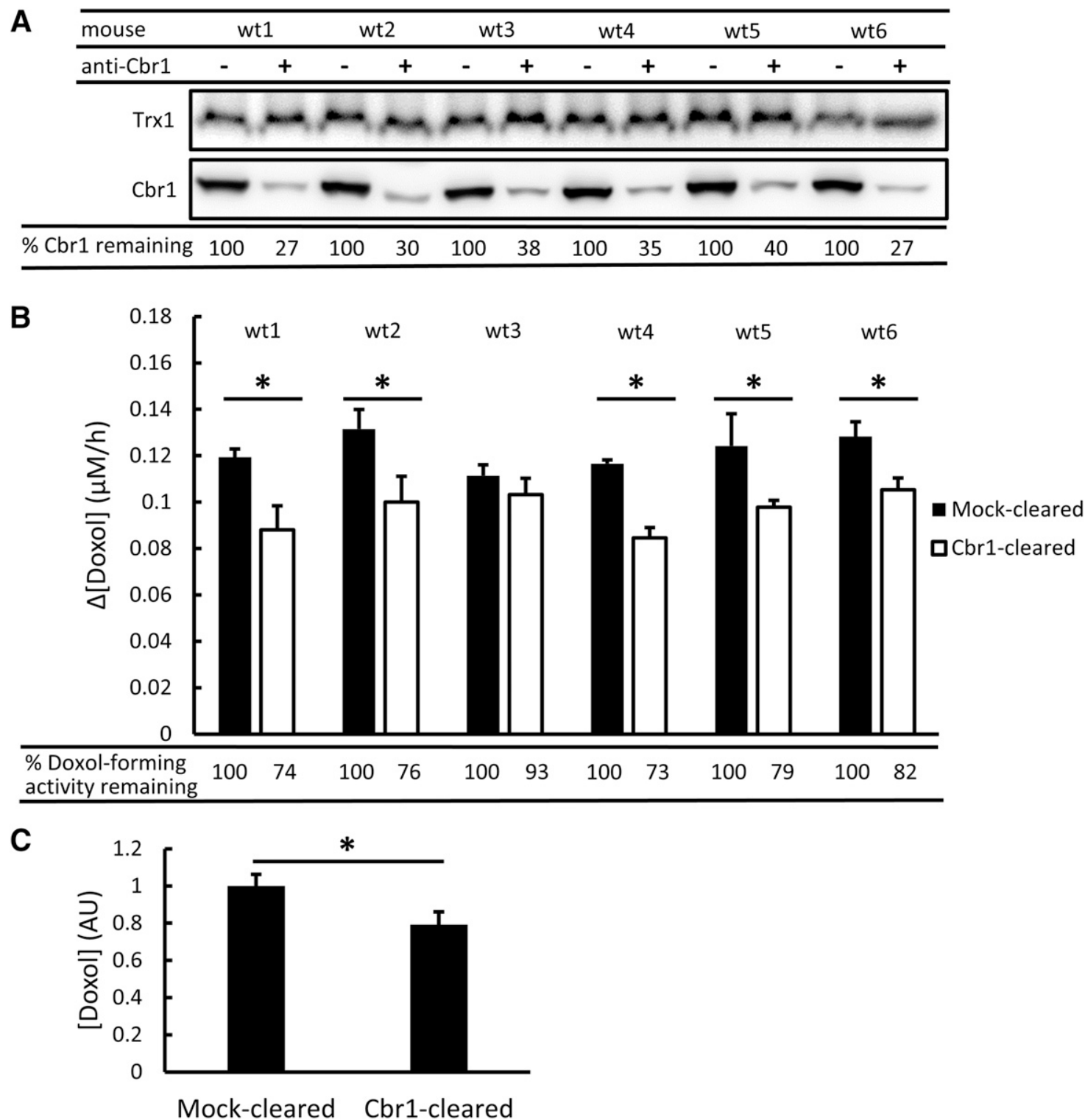

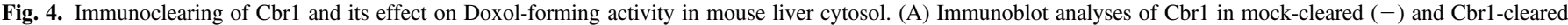

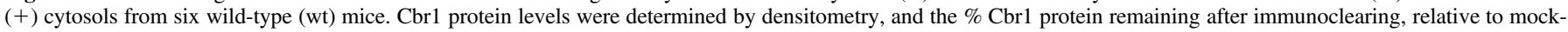

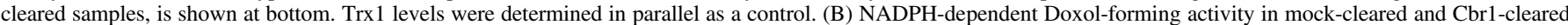

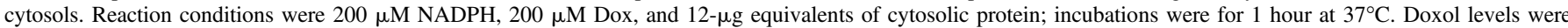

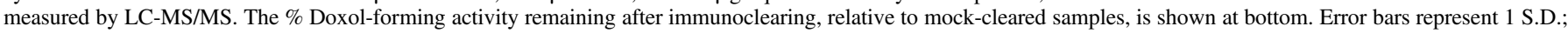

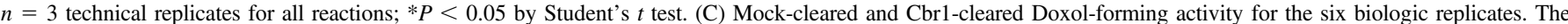

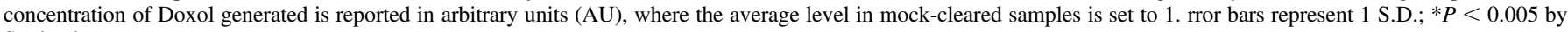
Student's $t$ test.

experiment that enzymes other than Cbr1 comprised the majority of the Doxol-forming activity in liver cytosol.

\section{Discussion}

Although several purified enzymes have been shown to catalyze Dox reduction, past efforts to test the biologic relevance of such findings have relied exclusively on pharmacological and genetic approaches. However, these approaches share the fundamental weakness of nonspecificity. Enzyme inhibitors rarely are specific for only one enzyme, and altering the genome for the purpose of over- or underexpressing a specific gene often has unpredictable effects on the expression of other genes. To avoid these issues, we developed a method of studying Doxol formation that takes advantage of the truly specific binding between antigen and antibody. We raised antibodies against Cbr1, the most studied Dox reductase, and against Cbr3 and Tr1, two other enzymes potentially involved in Dox reduction, and used the antibodies to immunoclear the respective proteins from mouse liver cytosol. By selectively removing each of these specific enzymes, we were able to quantify the contribution of each enzyme to Dox metabolism in the quasi in vivo context of liver cytosol.

Our initial characterization of the three enzymes revealed that $\mathrm{Cbr} 3$ and Cbr1 were both capable of producing Doxol but that Tr1 was not, despite efficiently catalyzing Dox-dependent NADPH oxidation. Further analyses revealed that purified Tr1 catalyzed Dox-dependent redox cycling, generating significant amounts of $\mathrm{H}_{2} \mathrm{O}_{2}$. However, subcellular fractionation analyses showed that Dox-dependent redox cycling activity in liver lysates was primarily microsomal, whereas $\operatorname{Tr} 1$ protein 


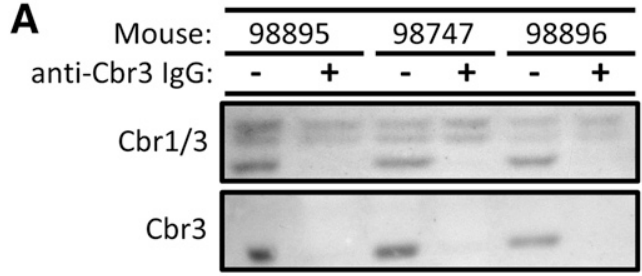

B
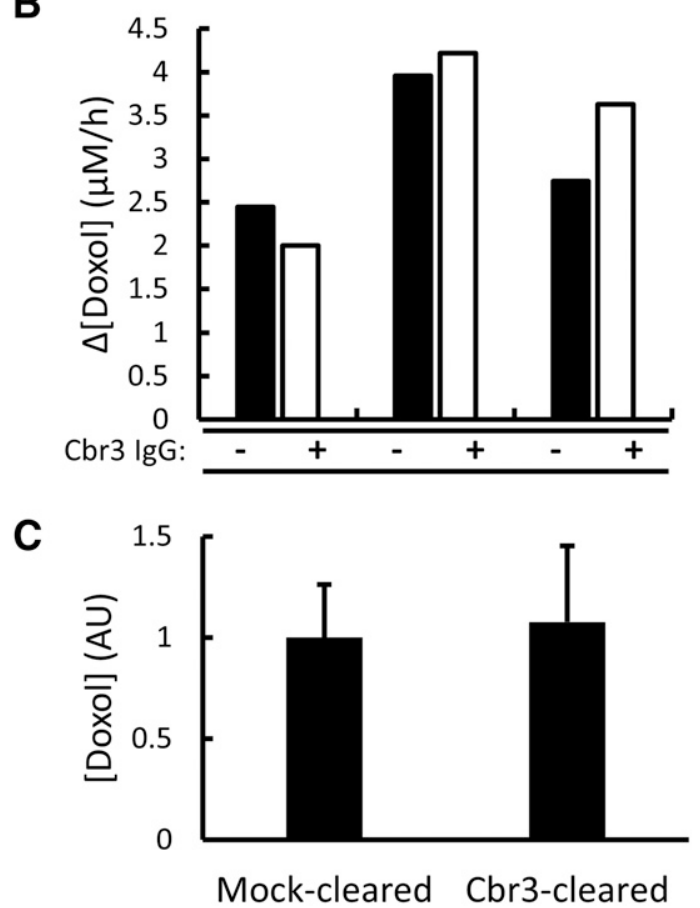
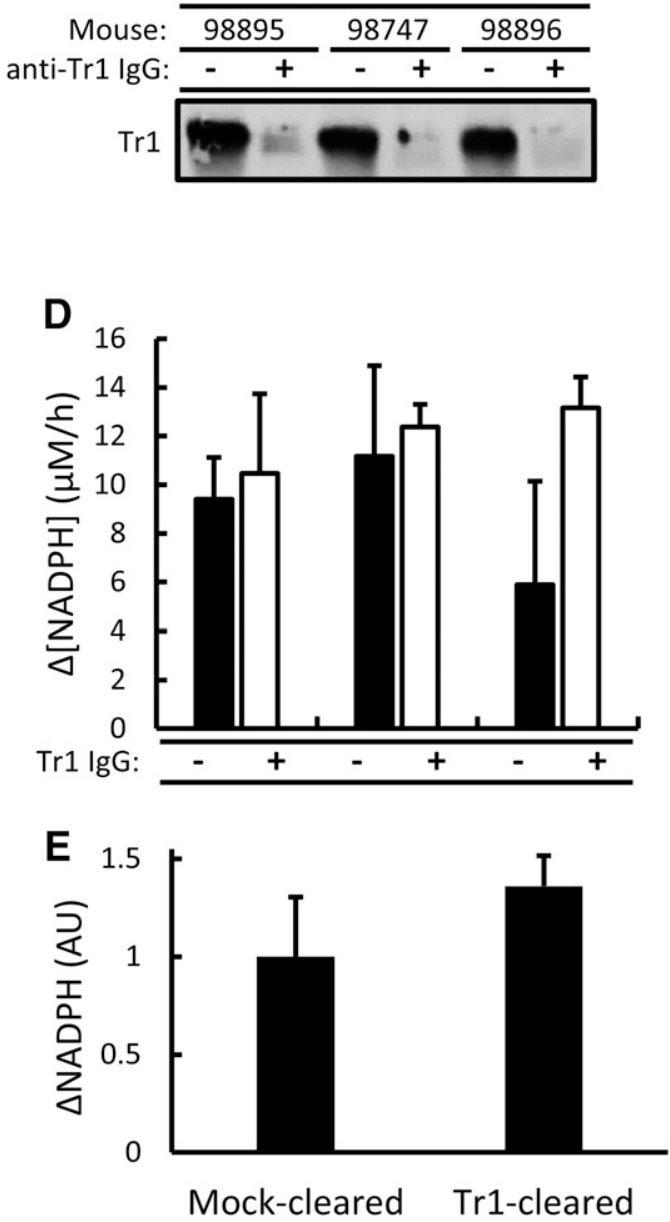

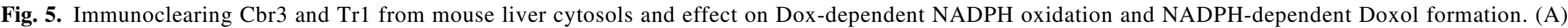

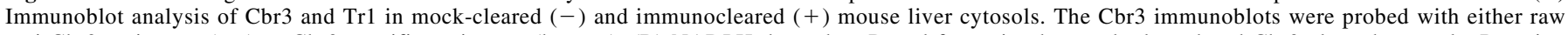

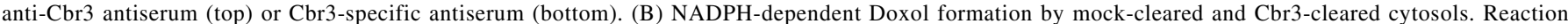

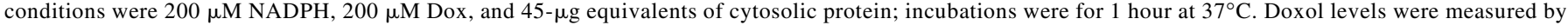

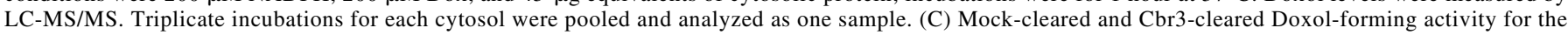

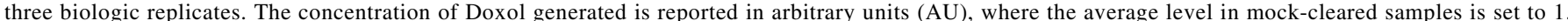

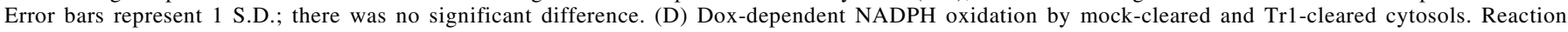

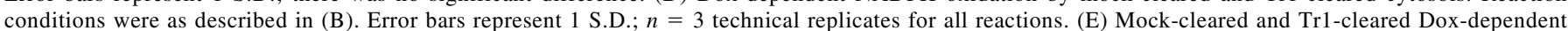

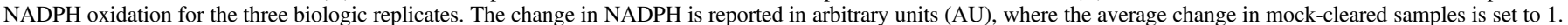
Error bars represent 1 S.D.; there was no significant difference.

was almost exclusively cytosolic. Furthermore, immunoclearing Tr1 from cytosol had no effect on the observed redox cycling activity. Tr1 was thus not a significant contributor to the Dox-dependent redox cycling activity observed in liver lysates. Identifying the Dox-dependent redox cycling activity is important because redox cycling may contribute to the chemotherapeutic and/or cardiotoxic activities of Dox. For example, Dox is known to intercalate into DNA, and if it were to participate in redox cycling reactions from that location, it could create localized high concentrations of DNA-damaging reactive oxygen species and thereby arrest replication.

When Cbr3 was immunocleared from liver cytosol, no reduction in Doxol-forming activity was observed, indicating that this enzyme did not contribute to Doxol formation by liver cytosol. In contrast, when Cbr1 was immunocleared from liver cytosol, $25 \%$ of the Doxol-forming activity was removed, indicating that $25 \%$ of the Doxol-forming activity in liver was attributable to the Cbr1 polypeptide. Importantly, $75 \%$ of the Doxol-forming activity in liver cytosol remained after Cbr1 was removed by immunoclearing, indicating that other enzymes constituted the majority of the Doxol-forming activity. This conclusion was further supported by ammonium sulfate fractionation results that showed that peak levels of Doxol-forming activity were detected in fractions that contained little Cbr1.

Cbr3 mRNA is the most highly induced message in both $\Delta T x n r d 1$ null (Bondareva et al., 2007; Suvorova et al., 2009) and $\Delta G c l m$ null tissues (Haque et al., 2010). We earlier suggested that Cbr3 played a role in Doxol formation based on the observations that cytosols from $\Delta G c l m$ livers and isolated hepatocytes had elevated levels of Cbr3 protein and elevated levels of Doxol-forming activity, that $\Delta G c l m$ hepatocytes incubated with Dox produced a substance toxic to myocytes and produced higher levels of Doxol, and that purified Cbr3 was able to reduce Dox to Doxol in vitro (Schaupp et al., 2015). Our current findings show that purified Cbr1 was 280-fold more efficient than Cbr3 in converting Dox to Doxol and that immunoclearing Cbr1, but not Cbr3, from either wild-type or $\Delta$ Txnrdl cytosols removed a significant fraction of the measurable Doxol-forming activity. In view of the current findings, we suspect that the increase in Doxolforming activity previously reported to occur in $\Delta G \mathrm{clm}$ livers (Schaupp et al., 2015) was due to induction of Cbr1, rather than induction of Cbr3. Cbrl is induced in both $\Delta G c l m$ and $\Delta T x n r d l$ livers, although not to the same extent as Cbr3. 
A

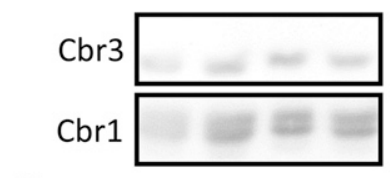

C

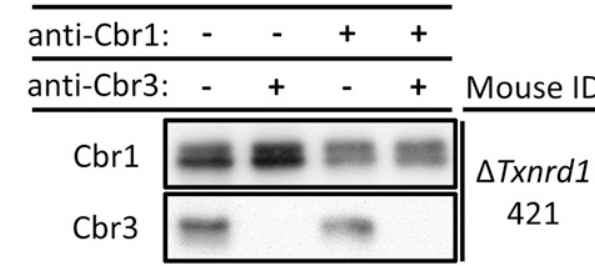

Cbr

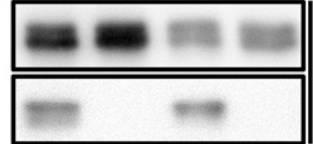

$\Delta T x n r d 1$

Cbr3

Cbr1

Cbr3

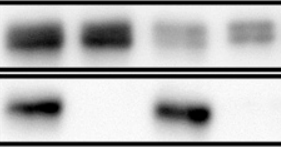

423

$\Delta T x n r d 1$

426

Cbr1

Cbr3

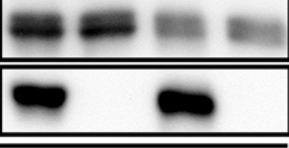

$\begin{array}{lllll}\text { \% Cbr1 protein } & 100 & 108 & 53 & 58\end{array}$

\begin{tabular}{lcccc} 
remaining: & $\pm 12 \quad \pm 14 \quad \pm 12$ \\
\hline$\%$ Cbr3 protein & 100 & 0 & 88 & 1
\end{tabular}

remaining:
B

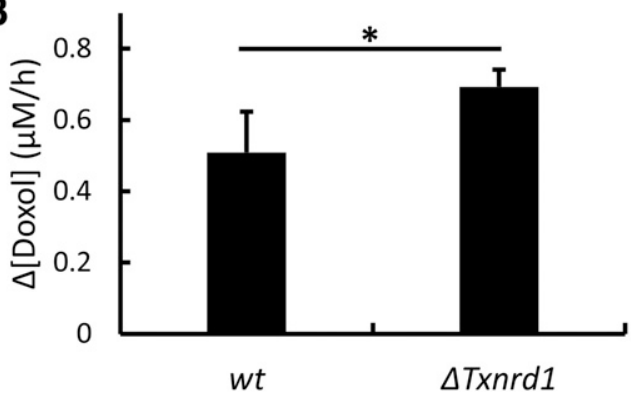

D

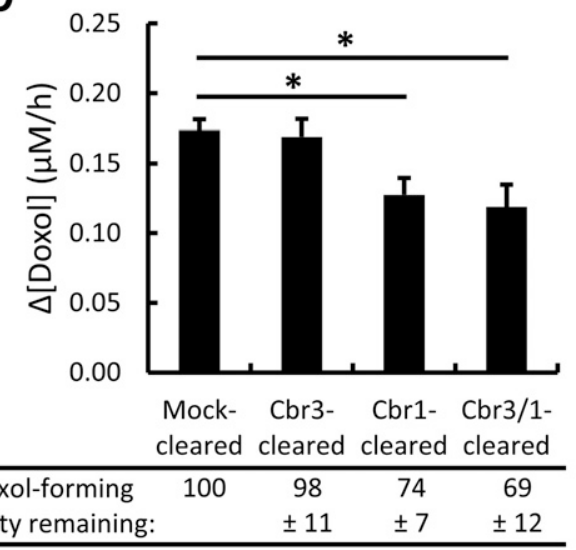

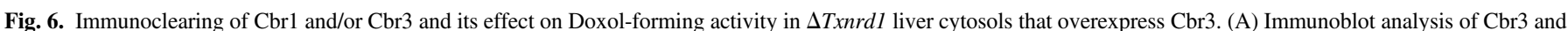

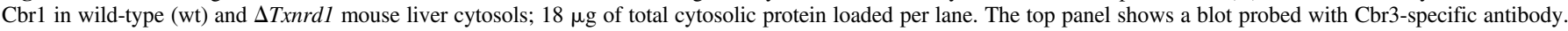

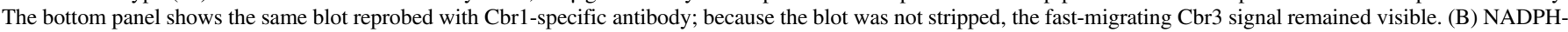

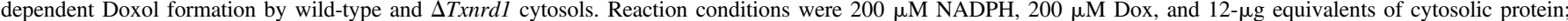

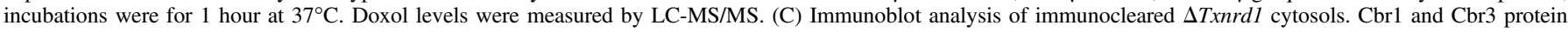

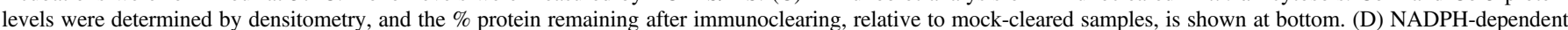

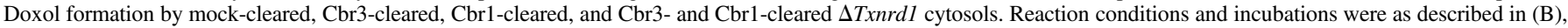

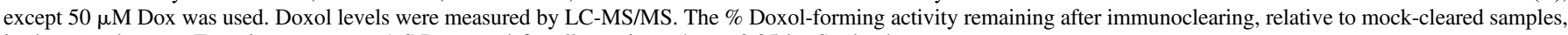
is shown at bottom. Error bars represent 1 S.D.; $n=4$ for all reactions; ${ }^{*} P<0.05$ by Student's $t$ test.

Olson et al. (2003) showed that mice systemically heterozygous for a $\triangle C b r l$ deletion mutation are more resistant to the cardiotoxic effects of Dox. This result suggested that halving the amount of Cbr1 in the cytosol was cardioprotective. As our results indicated that only $25 \%$ of the total Doxol-forming activity in wild-type cytosol was attributable to Cbr1, heterozygosity for the $\Delta C b r l$ null mutation would be expected to result in only a $12.5 \%$ reduction in total Doxol-forming activity. Thus, more experiments are necessary to understand the cardioprotective effect of $\triangle C b r 1$ heterozygosity during Dox treatment.

Others have shown (Jo et al., 2017) that coadministration of the Cbr1inhibiting drug 3-(7-isopropyl-4-(methylamino)-7H-pyrrolo[2,3-d]pyrimidin$5 y 1)$ phenol (hydroxy-PP-Me) during Dox treatment of rodents results in greater chemotherapeutic activity against implanted tumors and lower cardiotoxicity, and they concluded that Cbr1 plays a major role in converting Dox to cardiotoxic Doxol. Our current results are consistent with a role for Cbr1 in Doxol formation, but it is difficult to explain how inhibition of $\mathrm{Cbr} 1$ alone results in reduced cardiotoxicity when Cbr1 constitutes only $25 \%$ of the total Doxol-forming in liver cytosol. It is possible that Cbr1 constitutes a much higher percentage of total Doxol-forming activity in heart or other tissues, but we consider it more likely that the hydroxy-PP-Me is not specific for Cbr1 and instead inhibits a broader spectrum of NADPH-dependent oxidoreductases, including the unidentified enzymes responsible for $75 \%$ of the
Doxol-forming activity in liver cytosol. Nevertheless, the hydroxy-PP-Me study is extremely important because it suggests that development of drugs that inhibit Doxol-forming enzymes represents a promising strategy for combating cardiotoxicity during Dox chemotherapy.

Identification of the enzyme or enzymes that constitute the remaining unidentified Doxol-forming activity is important. Another enzyme capable of catalyzing Doxol formation in vitro is Akr1C3 (Kassner et al., 2008). However, the Doxol-forming activity of Akr1C3 is 10-fold more sensitive to the inhibitor 4-amino-1-tert-butyl-3-(2-hydroxyphenyl) pyrazolo[3,4-d]pyrimidine than the activity detected in cytosol (Tanaka et al., 2005; Kassner et al., 2008), reducing the likelihood that Akr1C3 plays a physiologic role in Doxol formation. Other aldo-keto reductases potentially play a role in Dox metabolism. For example, pharmacogenetic studies have suggested Akr1B10 or Akr1B1 may mediate the cardiotoxic effects of Dox (Morikawa et al., 2015; Sonowal et al., 2017). However, as the drugs used in these studies likely targeted other oxidoreductases, it is unclear whether the described effects were mediated by Akr1B10 or Akr1B1. The microsomal enzyme $11 \beta$-hydroxysteroid dehydrogenase 1 (11 $\beta$-HSD1) was also recently linked to Doxol formation (Yang et al., 2018). The study showed that Doxol formation in human liver hepatocytes is reduced by treatment with an inhibitor of $11 \beta$-HSD1. Because the inhibitor used was not specific to $11 \beta$-HSD1, inhibition of other oxidoreductases may have been responsible for the effect of the drug on 


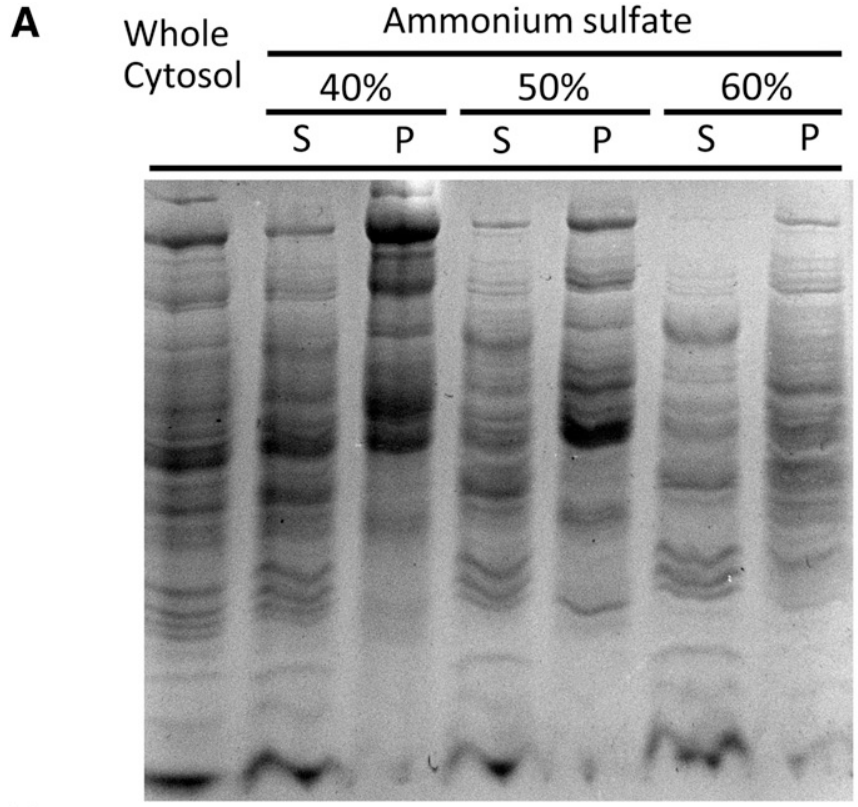

B
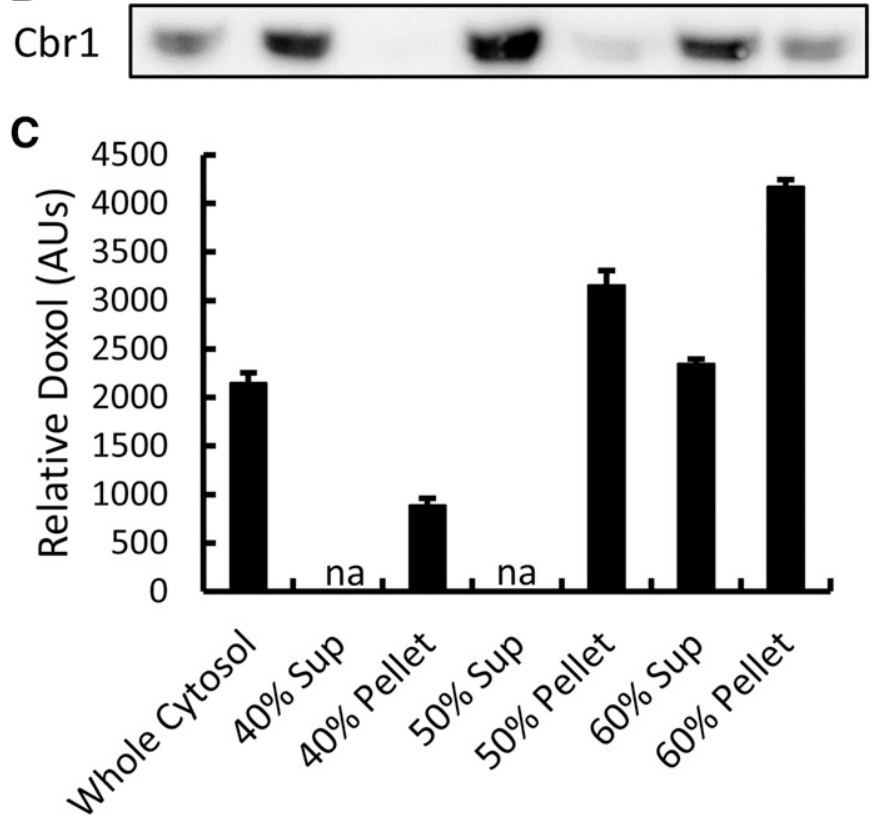

Fig. 7. Cbr1 levels and Doxol-forming activity in ammonium sulfate-fractionated mouse liver cytosol. (A) Coomassie-stained SDS-PAGE analysis of proteins in ammonium sulfate fractions; $10 \mu \mathrm{g}$ of protein loaded per lane. P, pellet; $\mathrm{S}$, supernatant. (B) Immunoblot analysis of Cbr1 in ammonium sulfate fractions; $10 \mu \mathrm{g}$ of protein loaded per lane. (C) Relative amount of Doxol produced by each fraction. Reaction conditions were $200 \mu \mathrm{M}$ NADPH, $200 \mu \mathrm{M}$ Dox, and $12 \mu \mathrm{g}$ fraction protein; incubations were for 1 hour at $37^{\circ} \mathrm{C}$. Doxol levels were determined by LC-MS/MS. The relative level of Doxol generated is reported in arbitrary units (AU), where the average level in whole cytosol is set to 2200. Error bars represent 1 S.D.; $n=3$ for all reactions; na, not assayed; sup, supernatant.

Doxol-forming activity in vivo. Furthermore, the current study showed Doxol formation to be predominantly cytosolic rather than microsomal. Moreover, although the inhibitor used was not specific for $11 \beta$-HSD1, it was shown to not inhibit Cbr1. Thus, the reduction of Doxol formation seen following treatment with the inhibitor is further evidence for the existence of enzymes other than Cbr1 that play an important role in Doxol formation.

The current study showed that although Cbr1 contributed significantly to Doxol formation in liver cytosol, currently unidentified enzyme or enzymes were responsible for the majority of the Doxol formation activity. The immunoclearing approach outlined here provides a method for evaluating the contributions of other potential Dox reductases such as AKR1C3 or $11 \beta$-HSD1. However, rather than evaluating specific candidate enzymes using an immunoclearing approach, identification of the enzyme(s) responsible for the unaccounted portion of hepatic Doxol formation likely will require exhaustive fractionation of cytosolic proteins to achieve purity, biochemical identification of specific proteins, and subsequent immunoclearing validation. The ammonium sulfate fractionation of mouse liver cytosol described in this study represents a potential first step in this process and demonstrates that fractionation and tracking Doxol-forming activity is a viable method for identifying unknown Dox reductases.

\section{Acknowledgments}

We thank Jeff Morre of the Oregon State University Mass Spectrometry Facility for assistance in developing the Doxol assay, Oregon State University undergraduates Brittany Marshal and Blake Migaki for assistance in constructing expression plasmids and fractionating liver cytosol, and University of Washington undergraduate Claire Chisholm for assistance in assaying protein levels. The primary laboratory was that of G.F.M.

\section{Authorship Contributions}

Participated in research design: Breysse, Schaupp, Kavanagh, Schmidt, G.F. Merrill.

Conducted experiments: Breysse, Boone, Long, M.E. Merrill, Schaupp, G.F. Merrill.

Contributed new reagents or analytic tools: White, Kavanagh, Schmidt, G.F. Merrill.

Performed data analysis: Breysse.

Wrote or contributed to the writing of the manuscript: Breysse, Boone, M.E. Merrill, G.F. Merrill.

\section{References}

Arnér ES, Nordberg J, and Holmgren A (1996) Efficient reduction of lipoamide and lipoic acid by mammalian thioredoxin reductase. Biochem Biophys Res Commun 225:268-274.

Bains OS, Karkling MJ, Lubieniecka JM, Grigliatti TA, Reid RE, and Riggs KW (2010) Naturally occurring variants of human CBR3 alter anthracycline in vitro metabolism. J Pharmacol Exp Ther 332:755-763.

Bains OS, Szeitz A, Lubieniecka JM, Cragg GE, Grigliatti TA, Riggs KW, and Reid RE (2013) A correlation between cytotoxicity and reductase-mediated metabolism in cell lines treated with doxorubicin and daunorubicin. J Pharmacol Exp Ther 347:375-387.

Bernardini N, Giannessi F, Bianchi F, Dolfi A, Lupetti M, Zaccaro L, Malvaldi G, and Del Tacca M (1991) Comparative activity of doxorubicin and its major metabolite, doxorubicinol, on V79/AP4 fibroblasts: a morphofunctional study. Exp Mol Pathol 55:238-250.

Björnstedt M, Hamberg M, Kumar S, Xue J, and Holmgren A (1995) Human thioredoxin reductase directly reduces lipid hydroperoxides by NADPH and selenocystine strongly stimulates the reaction via catalytically generated selenols. J Biol Chem 270:11761-11764.

Blanco JG, Leisenring WM, Gonzalez-Covarrubias VM, Kawashima TI, Davies SM, Relling MV, Robison LL, Sklar CA, Stovall M, and Bhatia S (2008) Genetic polymorphisms in the carbonyl reductase 3 gene CBR3 and the $\mathrm{NAD}(\mathrm{P}) \mathrm{H}$ :quinone oxidoreductase 1 gene NQO1 in patients who developed anthracycline-related congestive heart failure after childhood cancer. Cancer 112:2789-2795.

Bondareva AA, Capecchi MR, Iverson SV, Li Y, Lopez NI, Lucas O, Merrill GF, Prigge JR, Siders AM, Wakamiya M, et al. (2007) Effects of thioredoxin reductase-1 deletion on embryogenesis and transcriptome. Free Radic Biol Med 43:911-923.

Boucek RJ Jr, Olson RD, Brenner DE, Ogunbunmi EM, Inui M, and Fleischer S (1987) The major metabolite of doxorubicin is a potent inhibitor of membrane-associated ion pumps. A correlative study of cardiac muscle with isolated membrane fractions. J Biol Chem 262:15851-15856.

Chang BK, Brenner DE, and Gutman R (1989) Cellular pharmacology of doxorubicinol alone and combined with verapamil in pancreatic cancer cell lines. Anticancer Res 9:341-345.

Dodd DA, Atkinson JB, Olson RD, Buck S, Cusack BJ, Fleischer S, and Boucek RJ Jr (1993) Doxorubicin cardiomyopathy is associated with a decrease in calcium release channel of the sarcoplasmic reticulum in a chronic rabbit model. J Clin Invest 91:1697-1705.

Forrest GL, Gonzalez B, Tseng W, Li X, and Mann J (2000) Human carbonyl reductase overexpression in the heart advances the development of doxorubicin-induced cardiotoxicity in transgenic mice. Cancer Res 60:5158-5164.

Frezza C, Cipolat S, and Scorrano L (2007) Organelle isolation: functional mitochondria from mouse liver, muscle and cultured fibroblasts. Nat Protoc 2:287-295.

Hanna AD, Lam A, Tham S, Dulhunty AF, and Beard NA (2014) Adverse effects of doxorubicin and its metabolic product on cardiac RyR2 and SERCA2A. Mol Pharmacol 86:438-449.

Haque JA, McMahan RS, Campbell JS, Shimizu-Albergine M, Wilson AM, Botta D, Bammler TK, Beyer RP, Montine TJ, Yeh MM, et al. (2010) Attenuated progression of diet-induced steatohepatitis in glutathione-deficient mice. Lab Invest 90:1704-1717.

Heibein AD, Guo B, Sprowl JA, Maclean DA, and Parissenti AM (2012) Role of aldo-keto reductases and other doxorubicin pharmacokinetic genes in doxorubicin resistance, DNA binding, and subcellular localization. BMC Cancer 12:381. 
Jo A, Choi TG, Jo YH, Jyothi KR, Nguyen MN, Kim JH, Lim S, Shahid M, Akter S, Lee S, et al. (2017) Inhibition of carbonyl reductase 1 safely improves the efficacy of doxorubicin in breast cancer treatment. Antioxid Redox Signal 26:70-83.

Joerger M, Huitema AD, Meenhorst PL, Schellens JH, and Beijnen JH (2005) Pharmacokinetics of low-dose doxorubicin and metabolites in patients with AIDS-related Kaposi sarcoma. Cancer Chemother Pharmacol 55:488-496.

Kassner N, Huse K, Martin HJ, Gödtel-Armbrust U, Metzger A, Meineke I, Brockmöller J, Klein $\mathrm{K}$, Zanger UM, Maser E, et al. (2008) Carbonyl reductase 1 is a predominant doxorubicin reductase in the human liver. Drug Metab Dispos 36:2113-2120.

Kremer LC and Caron HN (2004) Anthracycline cardiotoxicity in children. $N$ Engl J Med 351:120-121.

Luthman M and Holmgren A (1982) Rat liver thioredoxin and thioredoxin reductase: purification and characterization. Biochemistry 21:6628-6633.

May JM, Mendiratta S, Hill KE, and Burk RF (1997) Reduction of dehydroascorbate to ascorbate by the selenoenzyme thioredoxin reductase. J Biol Chem 272:22607-22610.

Morikawa Y, Kezuka C, Endo S, Ikari A, Soda M, Yamamura K, Toyooka N, El-Kabbani O, Hara A, and Matsunaga T (2015) Acquisition of doxorubicin resistance facilitates migrating and invasive potentials of gastric cancer MKN45 cells through up-regulating aldo-keto reductase 1B10. Chem Biol Interact 230:30-39.

Olson LE, Bedja D, Alvey SJ, Cardounel AJ, Gabrielson KL, and Reeves RH (2003) Protection from doxorubicin-induced cardiac toxicity in mice with a null allele of carbonyl reductase 1 Cancer Res 63:6602-6606.

Olson RD, Mushlin PS, Brenner DE, Fleischer S, Cusack BJ, Chang BK, and Boucek RJ Jr (1988) Doxorubicin cardiotoxicity may be caused by its metabolite, doxorubicinol. Proc Natl Acad Sci USA 85:3585-3589.
Schaupp CM, White CC, Merrill GF, and Kavanagh TJ (2015) Metabolism of doxorubicin to the cardiotoxic metabolite doxorubicinol is increased in a mouse model of chronic glutathione deficiency: a potential role for carbonyl reductase 3. Chem Biol Interact 234:154-161.

Singal PK and Iliskovic N (1998) Doxorubicin-induced cardiomyopathy. N Engl J Med 339:900-905. Sonowal H, Pal PB, Wen JJ, Awasthi S, Ramana KV, and Srivastava SK (2017) Aldose reductase inhibitor increases doxorubicin-sensitivity of colon cancer cells and decreases cardiotoxicity. $S c i$ Rep 7:3182.

Suvorova ES, Lucas O, Weisend CM, Rollins MF, Merrill GF, Capecchi MR, and Schmidt EE (2009) Cytoprotective Nrf2 pathway is induced in chronically txnrd 1-deficient hepatocytes. PLoS One 4:e6158.

Swain SM, Whaley FS, and Ewer MS (2003) Congestive heart failure in patients treated with doxorubicin: a retrospective analysis of three trials. Cancer 97:2869-2879.

Tanaka M, Bateman R, Rauh D, Vaisberg E, Ramachandani S, Zhang C, Hansen KC, Burlingame AL, Trautman JK, Shokat KM, et al. (2005) An unbiased cell morphology-based screen for new, biologically active small molecules. PLoS Biol 3:e128.

Yang X, Hua W, Ryu S, Yates P, Chang C, Zhang H, and Di L (2018) 11ß-hydroxysteroid dehydrogenase 1 human tissue distribution, selective inhibitor, and role in doxorubicin metabolism. Drug Metab Dispos 46:1023-1029.

Address correspondence to: Gary F. Merrill, Department of Biochemistry and Biophysics, 2011 Ag Life Sci Bldg, 2750 Campus Way, Oregon State University, Corvallis, OR 97331. E-mail: merrillg@onid.orst.edu 